\title{
Robust multiple objects tracking using image segmentation and trajectory estimation scheme in video frames
}

\author{
Ying-Tung Hsiao ${ }^{\mathrm{a}}$, Cheng-Long Chuang ${ }^{\mathrm{b}}$, Yen-Ling Lu ${ }^{\mathrm{c}}$, Joe-Air Jiang ${ }^{\mathrm{b}, *}$ \\ ${ }^{a}$ Department \& Graduate School of Computer Science, National Taipei University of Education, Taipei, Taiwan, ROC \\ ${ }^{\mathrm{b}}$ Department of Bio-Industrial Mechatronics Engineering, National Taiwan University, No. 1, Section 4, Roosevelt Road, Taipei 106, Taiwan, ROC \\ ${ }^{\mathrm{c}}$ Information Office, Central Personnel Administration, Taipei, Taiwan, ROC
}

Received 28 February 2005; received in revised form 31 October 2005; accepted 9 April 2006

\begin{abstract}
In this paper, a novel image segmentation and a robust unsupervised video objects tracking algorithm are proposed. The proposed method is able to track complete object regions in a sequence of video frames. In this work, object tracking is achieved by analysing the movement of the contours with frame by frame in the video stream. The proposed algorithm involves with three major components for analysing the shapes and motions of the object in the video frames. First, a modified mathematical morphology edge detection algorithm is utilized to extract the contour features in the video frames. Then, a contour-based image segmentation algorithm is proposed and applied to the contour features for partitioning the predetermined target objects in the video frames. Finally, a trajectory estimation scheme is developed to handle the movements of the objects in the video frames. The proposed image segmentation algorithm is capable of automatically partitioning the predetermined objects. The proposed tracking algorithm is also robust against overlapping and videos acquired by non-stationary cameras. The experimental results show that the proposed algorithm can precisely partition and track the predetermined objects in video frames.
\end{abstract}

(C) 2006 Elsevier B.V. All rights reserved.

Keywords: Mathematical morphology; Edge detection; Image segmentation; Motion estimation

\section{Introduction}

Tracking multiple predetermined objects in a sequence of video frames is a challenging and active research area in computer vision and visual surveillance. Object segmentation and object tracking are techniques that have widely applied to many applications, such as object-based video coding, contentbased video indexing and retrieval, video surveillance and entertainment interactions in videos (e.g. MPEG-4 and MPEG-7) [1,2]. Many previously proposed approaches have been developed for applications of video tracking. Among these approaches, the most common method is the background subtraction method [3]. However, the background subtraction

\footnotetext{
Abbreviations $\ominus$, mathematical morphological erosion operator; $\oplus$, mathematical morphological dilation operator; $\circ$, mathematical morphological opening operator; • mathematical morphological closing operator; WTH, mathematical morphological white top-hat operator; BTH, mathematical morphological black top-hat operator; $\mathrm{k}^{\mathrm{TH}}$, mathematical morphological contrast enhancement operator.

* Corresponding author. Tel.: +88623366 5341; fax: +88622362 7620 .

E-mail address: jajiang@ntu.edu.tw (J.-A. Jiang).
}

method is only suitable for video that captured by a stationary camera. Therefore, when the camera is a non-stationary one, the performance of the background subtraction method is degraded and impracticable.

To cope with this problem, several advanced algorithms have been proposed. For example, Comaniciu et al. [4] used the mean-shift approach to compute the translation of a circular region. Jepson et al. [5] developed a probabilistic appearance model to compute the affine motion of the object for capturing the stable object features. Huang [6] proposed a colour-based approach to reduce the resolution such that the motion of the objects in real-time can be computed. However, these approaches are only capable of tracking the central point or the orientation of the object.

Tracking of the complete regions of the objects can be achieved by analysing the contour features in the video frames. For example, Yezzi et al. [7] combined the Mumford-Shah distance with 2D transformation for computing the contour transformation between two different views, but it requires a predetermined shape mask to perform object tracking. Rittscher et al. [8] tracked the objects using possible affine deformations. Patras et al. [9] used watershed approach and grouped homogeneous regions to semantic 
objects that require human supervises. However, these approaches are unable to accurately extract the user desired objects in the image or video frames, and the tracking performances might be limited.

In this work, a mathematical morphology-based approach is presented. The proposed algorithm is a hybrid of three major components, which are an enhanced mathematical morphological edge detector, a novel contour-based image segmentation algorithm, and a novel trajectory estimation scheme, respectively. The utilized edge detector is one of our earlier works; it is an enhanced version of the mathematical morphology edge detector, which is capable of sketching out more thin edge features in low contrast regions that ordinary be eliminated by other conventional edge detectors [10]. Moreover, the proposed image segmentation algorithm is also utilized to partition the user predetermined objects in the video frame. The proposed segmentation method is based on region growing and region merging technique $[11,12]$. It is capable of precisely partitioning the predetermined object in video frames. Since the regions of the objects are obtained in the video frame, the proposed trajectory estimation scheme is applied for estimating the positions of the predetermined objects in the following sequences of video frames. The conceptual block diagram is depicted in Fig. 1.

This paper is organized as follows: Section 2 briefly introduces mathematical morphology, its basic morphological operations, and the utilized edge detector. Section 3 proposes the region growing/merging-based image segmentation algorithm. Section 4 presents the proposed trajectory estimation algorithm. Experimental tacking results obtained by the proposed algorithm are presented and discussed in Section 5. Some comparison results between the proposed algorithm and some conventional algorithms are demonstrated in Section 6, and conclusions are given in Section 7.

\section{Mathematical morphological operators}

\subsection{General descriptions}

In this work, we utilized a mathematical morphological edge detector to sketch out the contour features in the video frames. Because the proposed tracking algorithm is based on the contour features in the video frames, edge features detection is an important task for the proposed algorithm to produce correct masks for extracting desired objects. Mathematical morphology theory was first invented by Matheron in the 1970s [13]. It was originally developed for analysing geometric structures. Serra was the first one who utilized the mathematical morphology theory to perform image analysis [13]. Mathematical morphology is the analysis of non-linear signals in terms of shapes. This simply means that morphology works by changing shape of objects contained within the nonlinear signals. The image is treated as a set in the operations based on the mathematical morphology, and the operands in all operations of the mathematical morphology are treated as set, as well. There are two sets of signals involved in the mathematical morphological operations. One is the shape signal that determined by the values that the signal takes on, and another is the shape of the signal that is extracted by applying a structure element to operate on the signal. Morphology can be applied to a wide range of applications, such as image enhancement, image restoration, edge detection, and noise reduction.

\subsection{Basic morphological operators}

There are two basic morphological operators: erosion and dilation. These operators are usually applied in tandem. Many other operations are derived from the operations defined in terms of erosion and dilation.

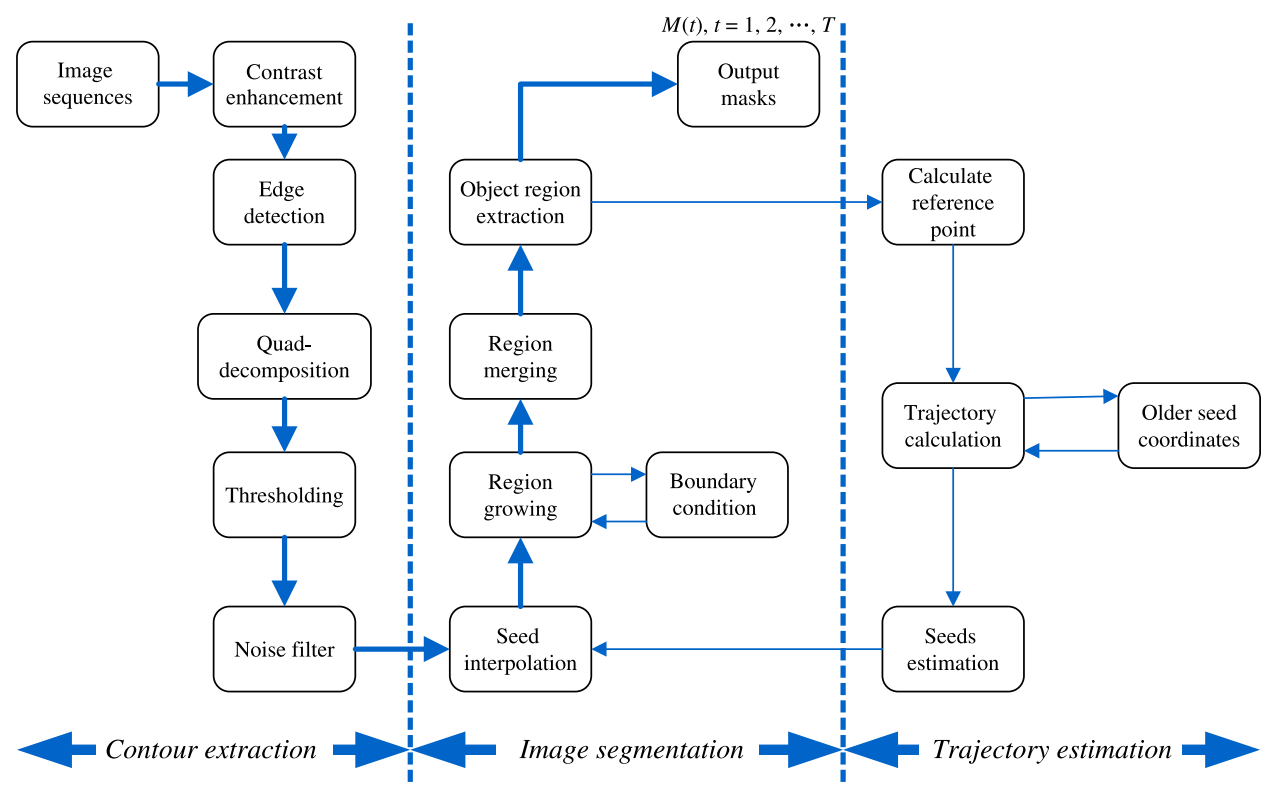

Fig. 1. Conceptual block diagram of the proposed algorithm. 
Erosion of a grey-level image $F$ by another structuring element $B$, denoted by $F \ominus B$, is defined as following:

$F \ominus B(x, y)=\min _{(i, j) \in B}\{F(x+i, y+j)\}$

where $(x, y)$ and $(i, j)$ are the coordinate sets of the image $F$ and the structuring element $B$, respectively. Erosion is a 'shrinking' operator. The values of $F \ominus B$ are always less than or equal to the values of $F$.

Dilation of a grey-level image $F$ by another structuring element $B$, denoted by $F \oplus B$, is defined as following:

$$
F \oplus B(x, y)=\min _{(i, j) \in B}\{F(x+i, y+j)\}
$$

Dilation is an 'expansion' operator. The values of $F \oplus B$ are always greater than or equal to the values of $F$.

By the erosion and dilation operators defined above, we can detect the edge in the image $F$, denoted by $E_{\mathrm{e}}(F)$, which is defined as the difference set of the original image $F$ and the erosion result of $F$. This is also known as erosion residue edge detector:

$E_{\mathrm{e}}(F)=F-(F \ominus B)$

Another method of detecting edge features in the image $F$, denoted by $E_{\mathrm{d}}(F)$, is defined as the difference set of the dilation result of $F$ and the original image $F$. This is also known as dilation residue edge detector:

$E_{\mathrm{d}}(F)=(F \oplus B)-F$

Opening of a data sequence $F$ by a structuring element $B$ is defined as erosion followed by dilation, which can be denoted by $F \circ B$ and expressed as following:

$F \circ B=(F \ominus B) \oplus B$

Opening of a data sequence can be interpreted as sliding the structuring element along the data sequence from beneath and the result is the highest points reached by any part of the structuring element. Generally, opening can smooth the sketch of an image and break narrow gaps.

Closing of a data sequence by a structuring element is defined as dilation followed by erosion, which can be denoted by $F \bullet B$ and expressed as following:

$F \bullet B=(F \oplus B) \ominus B$

Closing of a data sequence can be interpreted as sliding a 'flipped-over' version of the structuring element along the data sequence from above and the result is the lowest points reached by any part of the structuring element. Generally, closing can fuse narrow breaks and fill up small holes and gaps in the sketch.

The opening and closing operations can be used to construct top-hat transformation for enhancing the bright regions or dark regions. White top-hat transformation of image $F$, denoted as $\mathrm{WTH}(F)$, is defined as the difference set of the original image $F$ and the opening result of $F$ and can be formulated as follow:

$\mathrm{WTH}(F)=F-(F \circ B)$
Black top-hat transformation of image $F$, denoted as $\mathrm{BTH}(F)$, is defined as the summation set of the closing result of $F$ and the original image $F$ and can be formulated as follow:

$\operatorname{BTH}(F)=(F \bullet B)+F$

Therefore, a simple neighbourhood-based morphological contrast enhancement operation can be obtained by parallel computing the white and black top-hat of the image $F$. For example, the white top-hat can be added to the original image $F$ to enhance the bright objects and the black top-hat can be subtracted from resulting image to enhance the dark objects, respectively. The final top-hat contrast enhancement operator is denoted by $\kappa^{\mathrm{TH}}$ and can be formulated as follow:

$\kappa^{\mathrm{TH}}=F+\mathrm{WTH}(F)-\mathrm{BTH}(F)$

\subsection{Edge detector}

The primary goal of utilizing the edge detector [10] is to detect edge features in the video frames and preserve smooth contour features as well. In this section, the edge detector utilized in the proposed object tracking algorithm is briefly introduced. The overview conceptual block diagram of the edge detector is illustrated in Fig. 2.

The major objective of this step is to detect and preserve thin edge features in low contrast regions in the image. In this work, we utilized morphological top-hat contrast enhancement operator to increase the pixel intensity differences in the image. Therefore, we can obtain better quality of edge image using morphological dilation residue edge detector. However, when the thin edge features are in the places of low contrast regions, the strengths of pixel intensity differences are very weak and probably undetectable by a global threshold value.

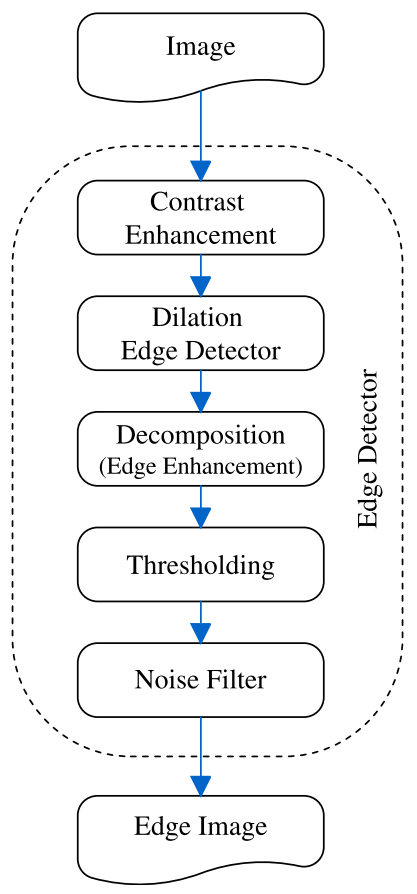

Fig. 2. Conceptual block diagram of the proposed morphological edge detection algorithm. 
Therefore, we divide the edge image into several sub-images using quad-decomposition technique, and enhance the pixel intensity differences in each sub-images by consulting the mean and standard deviation of the edge strength in the corresponding sub-image. Since the entire fine contour features are enhanced, a global threshold is then applied to transform the edge image into binary one. Moreover, five bipolaroriented edge masks are utilized to remove miss-detected edge points and reduce noise in the final image. The overall procedure of the proposed algorithm is illustrated in Fig. 3.

\subsubsection{Morphological contrast enhancement}

As mentioned in the last paragraph, the proposed algorithm consists of five steps. The first and second steps are based on the mathematical morphological operations. Let $F$ denote the original image set, and $B$ denote the structuring element set, and the formal descriptions are presented in the following paragraphs.

In order to increase the contrast level of the original image $F$, the morphological contrast enhancement process is applied to $F$ to increase the pixel intensity differences co-operating with a star structuring element $B$ as shown in Fig. 4 , and the contrast enhancement can be formulated as

$$
F_{\mathrm{c}}=F+\mathrm{WTH}(F)-\mathrm{BTH}(F)
$$

where $F_{\mathrm{c}}$ is the contrast enhanced image derived from the original image $F$, and the $\operatorname{WTH}(F)$ and $\operatorname{BTH}(F)$ are the white top-hat transformation and the black top-hat transformation of original image $F$, respectively. A simplified form of Eq. (10) can be derived from Eq. (7) to Eq. (9) as shown below:

$F_{\mathrm{c}}=2 F-(F \circ B)-(F \bullet B)$
The primary goal of the contrast enhancement process is to accentuate and sharpen the features in the image, and make the original image more useful for visualization for human eyes or let the image be analyzed by the following morphological dilation residue detector easier.

\subsubsection{Morphological dilation residue edge detector}

The morphological dilation residue edge detector is applied to the contrast enhanced image $F_{\mathrm{c}}$ to extract the contour feature details of the original image $F$. The process can be formulated as

$F_{\mathrm{e}}=\left(F_{\mathrm{c}} \oplus B\right)-F_{\mathrm{c}}$

where $F_{\mathrm{e}}$ is the extracted contour features that contains edges with various strengths.

Although the contrast level of the original image $F$ has been enhanced before detecting the edge features in the image, some smooth edges in the low contrast level regions are still too weak to be detected if a global threshold value is applied to $F_{\mathrm{e}}$ for extracting edge points. In order to prevent thin contour details from being eliminated by thresholding $F_{\mathrm{e}}$, it is necessary to develop a technique to enhance them. In Sections 2.3.3 and 2.3.4, a recursive quad-decomposition process and a set of edge enhancement cases are applied to the contour feature image $F_{\mathrm{e}}$ to increase the smooth edges in low contrast regions. Moreover, another set of bipolar oriented edge masks is also utilized to remove noise after thresholding process.

\subsubsection{Quad-decomposition edge enhancement}

As mentioned in Section 2.3.2, when level of brightness is not well-distributed in the entire original image $F$, the extracted contour features $F_{\mathrm{e}}$ might contains edges in various strengths.
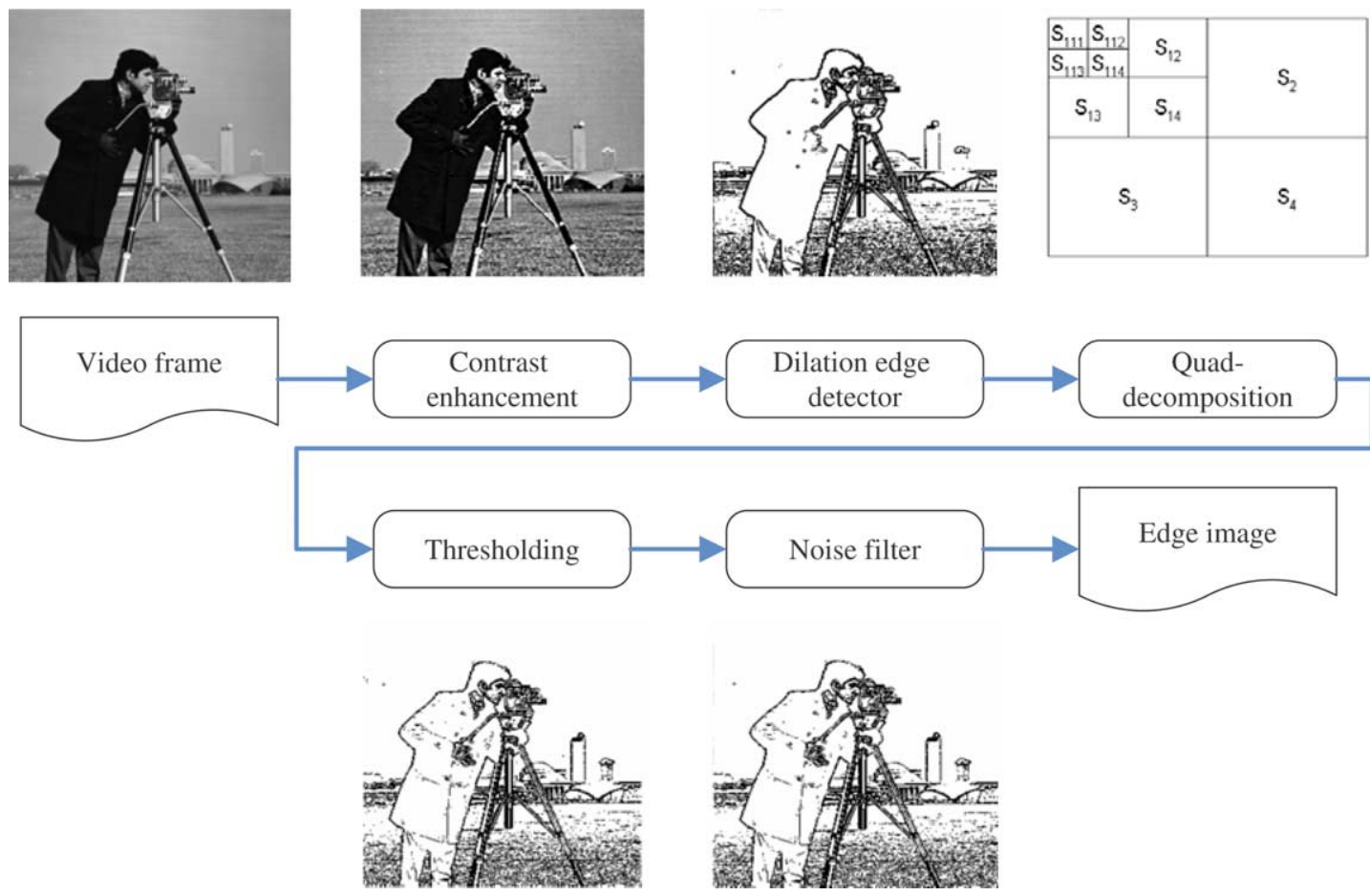

Fig. 3. Conceptual block diagram of the utilized edge detector. 


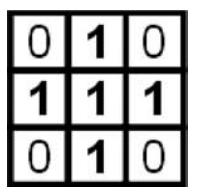

Fig. 4. Structuring element used in this work.

\begin{tabular}{|c|c|c|}
\hline$S_{111} S_{112}$ & \multirow{2}{*}{$S_{12}$} & \\
\cline { 1 - 1 }$S_{113}$ & $S_{114}$ & \\
\cline { 1 - 1 }$S_{13}$ & $S_{14}$ & \\
& & \\
\hline & & \\
& & \\
& & \\
& & \\
\end{tabular}

Fig. 5. Illustration of quad-decomposition of an image.

When we apply a global threshold value to $F_{\mathrm{e}}$, many contour features in regions too bright or too dark might be undetectable and be disappeared from the final edge extraction result. Therefore, enhancing some meaningful smooth edges is needed before thresholding $F_{\mathrm{e}}$. Hence, in this section, a new contour features enhancement strategy based on quaddecomposition technique is proposed and discussed as follows.

The quad-decomposition is a process that recursively decompose an image into four sub-image in equal size, as shown in Fig. 5. This process stops when side length of the subimage (width or height) reaches user-defined shortest side length. In this process, four edge point enhance cases are defined to distinguish sub-images that contain meaningful smooth edge features. However, before performing enhancement in each sub-image, the mean values and standard deviations of each sub-image have to be calculated first, which are

$\mu_{\alpha}=\frac{\sum_{i=1}^{M_{\alpha}} \sum_{j=1}^{N_{\alpha}} F_{\alpha}(i, j)}{M_{\alpha} \times N_{\alpha}}$

$\delta_{\alpha}=\sqrt{\frac{\sum_{i=1}^{M_{\alpha}} \sum_{j=1}^{N_{\alpha}}\left(F_{\alpha}(i, j)-\mu_{\alpha}\right)^{2}}{M_{\alpha} \times N_{\alpha}}}$

Table 1

Edge point enhancement cases

\begin{tabular}{lllll}
\hline Case & Condition & & & Update \\
\cline { 2 - 4 } & $\# 1$ & $\# 2$ & $\# 3$ \\
\hline Redundant background & $P_{\alpha}(i, j)<\mu_{\alpha}$ & $\mu_{\alpha}<2$ & $\delta_{\alpha}<2$ & $P_{\alpha}(i, j)=0$ \\
Thin edge enhancement & $P_{\alpha}(i, j)>\mu_{\alpha}$ and $P_{\alpha}(i, j)<\mu_{\alpha}+1.5 \times \delta_{\alpha}$ & $\mu_{\alpha}<2$ & $\delta_{\alpha}<2$ & $P_{\alpha}(i, j)=P_{\alpha}(i, j)^{2}$ \\
Intensive thin edge & $P_{\alpha}(i, j)>\mu_{\alpha}+1.5 \times \delta_{\alpha}$ and $P_{\alpha}(i, j)<\mu_{\alpha}+3 \times \delta_{\alpha}$ & $\mu_{\alpha}<2$ & $\delta_{\alpha}<2$ & $P_{\alpha}(i, j)=P_{\alpha}(i, j)^{3}$ \\
enhancement & & $\mu_{\alpha}>5$ & $\delta_{\alpha}>6$ & $P_{\alpha}(i, j)=P_{\alpha}(i, j)^{2}$ \\
Thin edge in complex & $P_{\alpha}(i, j)>\mu_{\alpha}+1.5 \times \delta_{\alpha}$ & & & \\
background & & &
\end{tabular}

where $F_{\alpha}$ is the $\alpha$ th sub-image obtained through dividing the extracted contour features $F_{\mathrm{e}} . M_{\alpha}$ and $N_{\alpha}$ are width and height of the sub-image $F_{\alpha}$, and $\mu_{\alpha}$ and $\delta_{\alpha}$ are mean and standard deviation of the sub-image $F_{\alpha}$, respectively.

According to $\mu_{\alpha}$ and $\delta_{\alpha}$ of each sub-image $F_{\alpha}$, one among four point enhancement cases is selected and applied to $F_{\alpha}$ to enhance the thin contour features in the corresponding region in $F_{\mathrm{e}}$. For every pixel $P_{\alpha}(i, j)$ in position of $(i, j)$ in sub-image $F_{\alpha}$ should be processed by the proposed edge point enhancement cases, which are summarized in Table 1 and defined in detail as follows.

2.3.3.1. Redundant background. For the pixel that its grey-level value $P_{\alpha}(i, j)$ is less than mean value $\mu_{\alpha}$ of associated sub-image $F_{\alpha}$, and the sub-image $F_{\alpha}$ with $\mu_{\alpha}<2$ and $\delta_{\alpha}<2$, then the pixel is considered to be background pixel, and will be pruned to grey-level 0 (i.e. black).

2.3.3.2. Thin edges enhancement. For the pixel that its greylevel value $P_{\alpha}(i, j)$ is greater than mean value $\mu_{\alpha}$ of its belonging sub-image $F_{\alpha}$ and less than $\mu_{\alpha}+1.5 \times \delta_{\alpha}$, and the sub-image $F_{\alpha}$ with $\mu_{\alpha}<2$ and $\delta_{\alpha}<2$, then the pixel is considered to be thin edge point, and will be enhanced to square of $P_{\alpha}(i, j)$ (i.e. $\left.P_{\alpha}(i, j)^{2}\right)$.

2.3.3.3. Intensive thin edges enhancement. For the pixel that its grey-level value $P_{\alpha}(i, j)$ is greater than $\mu_{\alpha}+1.5 \times \delta_{\alpha}$ and less than $\mu_{\alpha}+3 \times \delta_{\alpha}$, and its associated sub-image $F_{\alpha}$ with $\mu_{\alpha}<2$ and $\delta_{\alpha}<2$, then the pixel is considered to be intensive thin edge point, and will be enhanced to cube of $P_{\alpha}(i, j)$ (i.e. $\left.P_{\alpha}(i, j)^{3}\right)$.

2.3.3.4. Thin edges in complex background enhancement. For the pixel whose grey-level value $P_{\alpha}(i, j)$ is greater than $\mu_{\alpha}+$ $1.5 \times \delta_{\alpha}$, and its associated sub-images $F_{\alpha}$ with $\mu_{\alpha}>5$ and $\delta_{\alpha}>$ 6 , then the pixel is considered to be thin edge point in a complex background, and will be enhanced to square of $P_{\alpha}(i, j)$ (as $P_{\alpha}(i, j)^{2}$ ).

After all sub-images $F_{\alpha}$ have been processed through the edge point enhancement process, these sub-images are combined together in order of their decomposed sequence. Hence, the thin contour features are enhanced. The produced edge feature enhanced image is denoted by $F_{q}$, and then edge points can be obtained by thresholding $F_{q}$, discussed in Section 2.3.4. 


\subsubsection{Bipolar-oriented edge masks}

Before we apply the edge masks to the image $F_{q}$ for removing the noise points that created by procedures described in Section 2.3.3.4, we have to thresholding entire image by a global threshold value to transform $F_{q}$ into a binary image. The thresholding process can be described as

$F_{t}(i, j)= \begin{cases}0, & \text { if } F_{q}(i, j)<T \\ 1, & \text { if } F_{q}(i, j) \geq T\end{cases}$

where $F_{t}$ is the binary edge image, and $T$ is the given threshold value. A proper threshold value can be obtained experimentally. A greater threshold value can extract more thin edges, but also lead to extract more noise points that do not really belong to any edge. A smaller threshold value is still able to extract the enhanced thin edges without producing a huge amount of noise, but it may eliminate some of really thin edges away.
In this paper, we suggest to apply a threshold value ranging from 20 to 30 for producing the image $F_{t}$ with the thin edges clearly and without suffering from too many noise points.

Five bipolar-oriented edge masks, as shown in Fig. 6, are applied to the edge image $F_{t}$. The primary goal of applying these edge masks is to remove undesired noise pixels in $F_{t}$. The masks are only applied to pixels that satisfy the following terms:

(1) The pixel is an edge point.

(2) The associated sub-image $F_{\alpha}$ that the values of its mean and standard deviation obtained in Section 2.3.3 satisfies $\mu_{\alpha}<3$ and $\delta_{\alpha}<3$. This indicates that the sub-image belongs to background or low contrast regions in the original image $F$.

The scores of each mask applied to the pixel can be obtained by summing the products of the mask elements and the corresponding covered pixels in edge image $F_{t}$. If one of the

$$
\left[\begin{array}{ccccccc}
-2 & -2 & -2 & -2 & -2 & -2 & -2 \\
-2 & -2 & -2 & -2 & -2 & -2 & -2 \\
1 & 1 & 1 & 1 & 1 & 1 & 1 \\
2 & 2 & 2 & -2 & 2 & 2 & 2 \\
1 & 1 & 1 & 1 & 1 & 1 & 1 \\
-2 & -2 & -2 & -2 & -2 & -2 & -2 \\
-2 & -2 & -2 & -2 & -2 & -2 & -2
\end{array}\right]\left[\begin{array}{ccccccc}
-2 & -2 & 1 & 2 & 1 & -2 & -2 \\
-2 & -2 & 1 & 2 & 1 & -2 & -2 \\
-2 & -2 & 1 & 2 & 1 & -2 & -2 \\
-2 & -2 & 1 & -2 & 1 & -2 & -2 \\
-2 & -2 & 1 & 2 & 1 & -2 & -2 \\
-2 & -2 & 1 & 2 & 1 & -2 & -2 \\
-2 & -2 & 1 & 2 & 1 & -2 & -2
\end{array}\right]
$$

(a)

(b)

$$
\left[\begin{array}{ccccccc}
-1 & 1 & 2 & 1 & -2 & -2 & -2 \\
-1 & 1 & 2 & 1 & -1 & -2 & -2 \\
-1 & 1 & 2 & 1 & -1 & -2 & -2 \\
-2 & -1 & 1 & -2 & 1 & -1 & -2 \\
-2 & -2 & -1 & 1 & 2 & 1 & -1 \\
-2 & -2 & -1 & 1 & 2 & 1 & -1 \\
-2 & -2 & -2 & 1 & 2 & 1 & -1
\end{array}\right]\left[\begin{array}{ccccccc}
2 & 1 & -1 & -2 & -2 & -2 & -2 \\
1 & 2 & 1 & -1 & -2 & -2 & -2 \\
-1 & 1 & 2 & 1 & -1 & -2 & -2 \\
-2 & -1 & 1 & -2 & 1 & -1 & -2 \\
-2 & -2 & -1 & 1 & 2 & 1 & -1 \\
-2 & -2 & -2 & -1 & 1 & 2 & 1 \\
-2 & -2 & -2 & -2 & -1 & 1 & 2
\end{array}\right]
$$

(c)

$$
\left[\begin{array}{ccccccc}
-2 & 1 & 1 & 2 & 1 & -2 & -2 \\
1 & 1 & 1 & 2 & 1 & -2 & -2 \\
1 & 1 & 1 & 2 & 1 & -2 & -2 \\
2 & 2 & 2 & -2 & 1 & -2 & -2 \\
1 & 1 & 1 & 1 & 1 & -2 & -2 \\
-2 & -2 & -2 & -2 & -2 & -2 & -2 \\
-2 & -2 & -2 & -2 & -2 & -2 & -2
\end{array}\right]
$$

(d)

(e)

Fig. 6. Bipolar oriented edge masks. (a) For angel 0, (b) for angel $\pi / 2$, (c) for angel $3 \pi / 8$, and $5 \pi / 8$, (d) for angel $\pi / 4$ and $3 \pi / 4$, (e) for corner edge. 
scores is positive, then the corresponding pixel is considered to be a meaningful edge point; otherwise, the pixel is a noise and should be removed from edge image $F_{t}$.

By applying these bipolar-oriented edge masks, noise points in image $F_{t}$ can be easily removed, and the final edge image $F_{\mathrm{f}}$ produced. In $F_{\mathrm{f}}$, pixel values equal to 1 are edge points, otherwise are not. In order to clearly indicate the extracted edge, experimental results shown in the Section 5 are inversed intentionally.

\section{Region growing/merging-based image segmentation}

\subsection{General descriptions}

Since the contour features in the video frame $F$ is extracted, the proposed image segmentation algorithm is then applied to the corresponding edge image $F_{t}$. The proposed image segmentation algorithm is based on region growing [11] and region merging [12] methods. The objective of the proposed image segmentation algorithm is to partition the domain $R$ of the video frame $F$ into regions $\left\{R_{i}, i=1, \ldots, m\right\}$ based on the edge image $F_{t}$ corresponding to original video frame $F$.

The proposed image segmentation algorithm consists of four major steps, which are deployment of growing seeds, two stages of region growing process, and region merging process, respectively. These steps are illustrated in Fig. 7. The detailed operations of these steps are discussed in Sections 3.2 to 3.4, respectively.

\subsection{Deployment for growing seeds}

Tracking in a sequence of video flames is to find the region masks that the sizes and shapes are exactly the same as the desired tracking objects in the sequence of video frames. In this work, the coordinates of the initial growing seeds in the first two frames must be defined by users. The proposed algorithm can extract the user desired tracking objects automatically.

The growing seeds can be deployed at any positions in the region of the object that we desired to partition and track. For more amount of growing seeds deployed in the same region of the object, the region masks can be produced more precisely. But single growing seed for one region of object is already enough for the proposed algorithm to produce a great result of partitioning. An example of deploying growing seeds into the edge image $F_{t}$ is given in Fig. 8. The example edge image $F_{t}$ is depicted in Fig. 8(a), and two growing seeds are deployed in two regions of separate objects as shown in Fig. 8(b). Then, the seed starts to expand itself by aggregating 8-neighbor pixels that share an edge or a vertex with the pixels in the region of the seed, as depicted in Fig. 8(c). The detailed descriptions regarding region growing process are discussed in Section 3.3.

\subsection{Region growing process-first stage}

The process of region growing consists of two separate stages. In the first stage, the growing seeds expand its regions by aggregating its eight-neighbor empty pixels. The growing

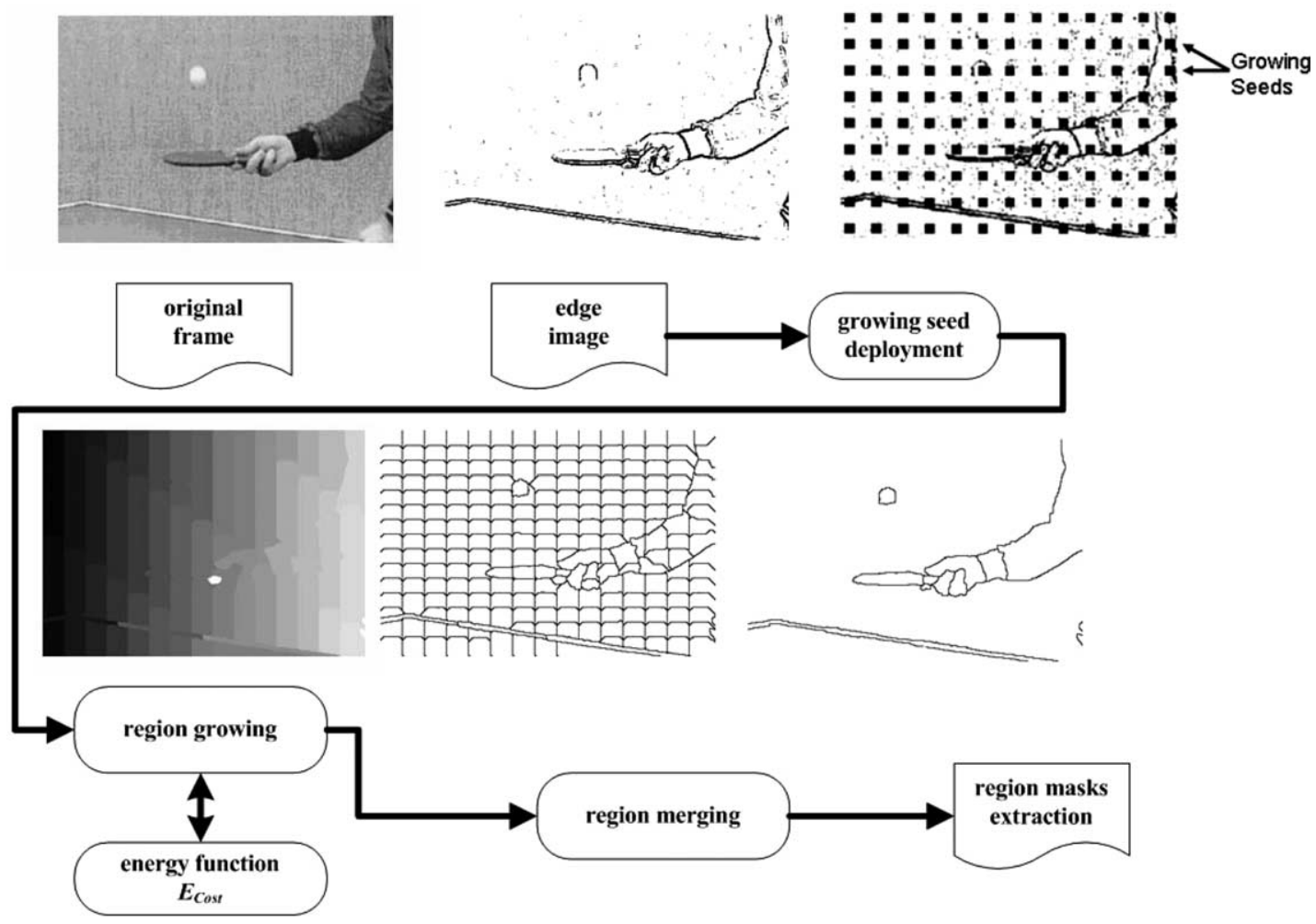

Fig. 7. Conceptual block diagram of the proposed image segmentation algorithm. 


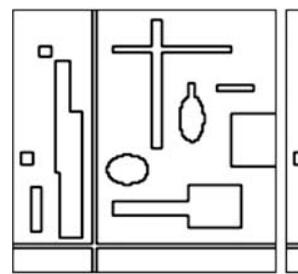

(a)

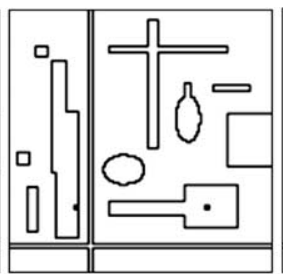

(b)

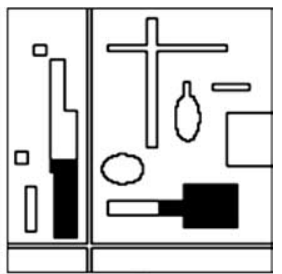

(c)
Fig. 8. (a) An example edge image $F_{t}$, (b) two growing seeds deployed in two regions of different objects, and (c) the result of region growing process after 35 iterations.

process in the first stage is controlled by an energy function. When a region of the $\lambda$ th seed, that its boundary pixels can be treated as a set $s_{\lambda}$, is trying to aggregate an empty pixel $\tau$, the energy function is evaluated to determine whether the pixel $\tau$ is appropriate to be aggregated by the set $s_{\lambda}$ or not. The pixel $\tau$ is temporary assumed to be an element in the set $s_{\lambda}$ as ${ }^{\prime} s_{\lambda}(i)$, where $' s_{\lambda}(i)$ is the new set. The energy function $E_{\mathrm{cost}}\left(s_{\lambda}(i)\right)$ is performed to evaluate the cost of the boundary elements between ' $s_{\lambda}(i-l)$ and ' $s_{\lambda}(i+l)$, where $l$ is the distance parameter. Therefore, the energy function $E_{\text {cost }}\left(s_{\lambda}(i)\right)$ can be formulated as follow:

$E_{\text {cost }}\left({ }^{\prime} s_{\lambda}(i)\right)=\sum_{x=i-l}^{i+l}\left(\alpha E_{\mathrm{e}}(x)+\beta E_{\mathrm{r}}(x)\right)-\gamma\left\|^{\prime} s_{\lambda}\right\|$

where $E_{\mathrm{e}}(x)$ and $E_{\mathrm{r}}(x)$ are shape cost and reached cost at the $x$ th position of the boundary of the $\lambda$ th region, $\left\|^{\prime} s_{\lambda}\right\|$ is the total amount of the pixels in the region $s_{\lambda}$, and $\alpha, \beta$ and $\gamma$ are parameters defined to control the importance $E_{\mathrm{e}}(x), E_{\mathrm{r}}(x)$ and $\left\|^{\prime} s_{\lambda}\right\|$, respectively. The edge shape cost and reached edge cost at the $x$ th position can be defined as follow:

$$
\begin{aligned}
E_{\mathrm{e}}(x)= & \eta_{1}\left|{ }^{\prime} s_{\lambda}(x)-{ }^{\prime} s_{\lambda}(x-1)\right|^{2} \\
& +\left.\eta_{2}\right|^{\prime} s_{\lambda}(x-1)-2^{\prime} s_{\lambda}(x)+\left.{ }^{\prime} s_{\lambda}(x+1)\right|^{2}
\end{aligned}
$$

$E_{\mathrm{r}}(x)=-F_{t}\left({ }^{\prime} s_{\lambda}(x)\right)$

where $\eta_{1}$ and $\eta_{2}$ are parameters defined to control the importance of the membrane and thin-plate term for the $x$ th boundary element, and $F_{t}\left(s_{\lambda}(x)\right)$ is the value in the position of the corresponding coordinate of ${ }^{\prime} s_{\lambda}(x)$ in the edge image $F_{t}$. By applying Eq. (16), the cost of aggregating the empty pixel $\tau$ can be obtained as $E_{\tau}$.

In order to measure the quality of the cost $E_{\tau}$, another cost $\neg E_{\tau}$, is evaluated. First, the pixel $\tau$ is removed from the set ${ }^{\prime} s_{\lambda}$ (the left set is the same as the original set $s_{\lambda}$ ) and then evaluates the cost $\neg E_{\tau}$ by the same method shown in Eq. (16), but the center point ${ }^{\prime} s_{\lambda}(i)$ in Eq. (16) is replaced by a boundary element in the set $s_{\lambda}$ that has the shortest distance between the pixel $\tau$ and itself. The decision of aggregating the pixel $\tau$ is based on the following rule:

$$
\begin{cases}\text { if } \quad & E_{\tau} \leq \neg E_{\tau} \rightarrow s_{\lambda}={ }^{\prime} s_{\lambda} \\ & \text { and delete unnecessary elements } \\ \text { if } \quad & E_{\tau}>\neg E_{\tau} \rightarrow s_{\lambda}=s_{\lambda}\end{cases}
$$

By utilizing the rule in (19), the region is attracted to grow to the boundary with the lowest cost. Therefore, the boundary in the set $s_{\lambda}$ shall converge on a set of meaningful edge points in $F_{t}$, and the first stage of region growing process is accomplished.

\subsection{Region growing process-second stage}

Since the first stage of region growing process is completed, the second stage follows the result that is produced by first stage, and lets the regions of seeds keep growing. The difference between the first stage and the second stage is that the second stage allows the growing regions to aggregate its eight-neighbor edge pixels without evaluating the energy function $E_{\text {cost }}$ in Eq. (16). For example, Fig. 9(a) shows the growing results of the first stage, in which the image consists of four individual regions and a non-continuous edge. In the second stage, the growing regions are allowed to aggregate the eight-neighbor edge pixels in the Fig. 9(a). The growing result of second stage is shown in Fig. 9(b), and the second stage of the region growing process is the finished.

\subsection{Region merging process}

The process of region merging is aimed to reduce redundant regions that do not have enough ratios of edge points between them. For a couple of regions, if the number of the shard edge pixels is smaller than $10 \%$ of the length of the shard region boundary, which is as shown in Fig. 9(c), then these two regions can be considered as homogeneous region and merged into a single region. After the process of region merging is completed, the segmentation line can be extracted as shown in Fig. 9(d), and can be used to extract different objects as shown in Fig. 9(e) and (f).

By employing the region growing and region merging techniques, we can partition the video frame into several meaningful components, and the process is robustness to noise

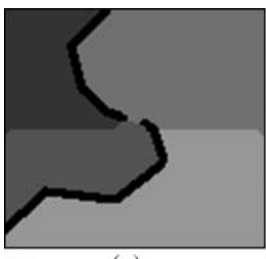

(a)

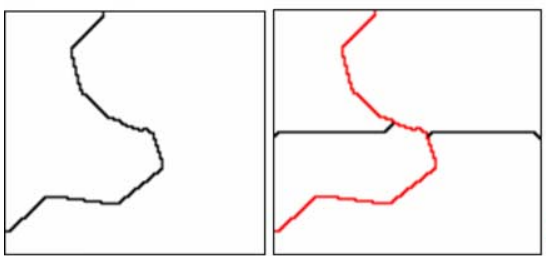

(d)

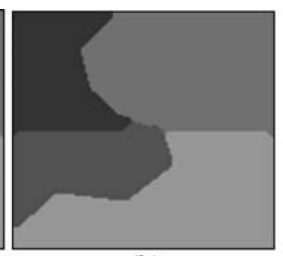

(b)

(e)

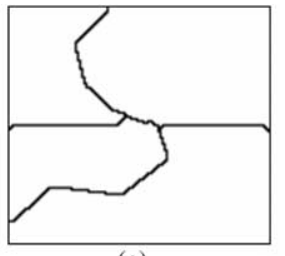

(c)

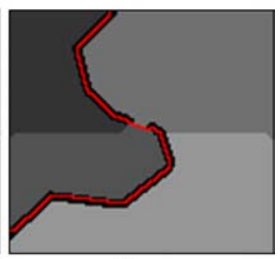

(f)
Fig. 9. (a) Result of the first stage of region growing process, (b) result of the second stage of region growing process, (c) edge lines of the regions obtained from the region growing process, (d) edge line of the result of the region merging process, (e) final edge line (red) overlays with (c), (f) final edge line (red) overlays with (a). 
pixels and the broken edges as well. An example of the entire partition process of a video frame has been embedded in Fig. 7, as well.

\section{Trajectory estimation scheme}

In this work, the primary objective is aimed to consecutively generate region masks for extracting the desired tracking objects with frame by frame in the video stream. According to the image segmentation algorithm proposed in Section 3, deploying a growing seed into the edge image $F_{t}$ is a necessary step to produce a region mask for the desired tracking object in the video frame $F$. Therefore, to develop a trajectory estimation scheme is needed for performing automatic growing seeds deployment. For cooperating with the proposed image segmentation algorithm, a trajectory estimation scheme is presented in this section.

The principle of the proposed trajectory estimation scheme is quite simple. First, the average coordinates $X_{O}(t)$ and $Y_{O}(t)$ of an object $O$ in the $t$ th video frame $F(t)$ are calculated as follow

$$
\begin{aligned}
& X_{O}(t)=\left(\sum_{p \subset S_{O}} x_{p}\right) /\left\|p \subset S_{O}\right\| \\
& Y_{O}(t)=\left(\sum_{p \subset S_{O}} y_{p}\right) /\left\|p \subset S_{O}\right\|
\end{aligned}
$$

where $p$ is the pixel in the video frame $F(t), S_{O}$ is the pixel set of object $O$, and $x_{p}$ and $y_{p}$ are the coordinates of pixel $p$ on $x$ - and $y$-axes, respectively. And the velocities $V x_{O}(t)$ and $V y_{O}(t)$ of object $O$ in $x$ axis- and $y$ axis-direction are obtained by the difference of average coordinates between the $(t-1)$ th and $t$ th video frames as follow:

$V x_{O}(t)=X_{O}(t-1)-X_{O}(t)$

$V y_{O}(t)=Y_{O}(t-1)-Y_{O}(t)$

Assume that the object $O$ is the grey area shown in Fig. 10 . After the moving velocity of the object $O$ in the $t$ th video frame $F(t)$ is obtained, the velocity information can be used to determine the position the initial growing seed for partitioning the same object $O$ in $(t+1)$ th video frame. In order to estimate a proper position for partitioning the same object $O$ in $(t+1)$ th video frame, the object $O$ segmented in th video frame is partitioned into four parts, which are left-top (LT) part, leftbottom (LB), right-top (RT), and right-bottom (RB) parts, respectively. Three parts among the four partitioned parts are selected such that the maximum area by summing up those of them can be achieved, and the corner part of the selected three parts is used to calculate the basis coordinate. The basis coordinate is the position of the initial growing seed for segmenting the same object $O$ in the $(t+1)$ th video frame. The basis coordinates $X_{B_{-}} O(t)$ and $Y_{B_{-}} O(t)$ for segmenting the object $O$ on $x$ - and $y$-axes in $(t+1)$ th video frame are defined as

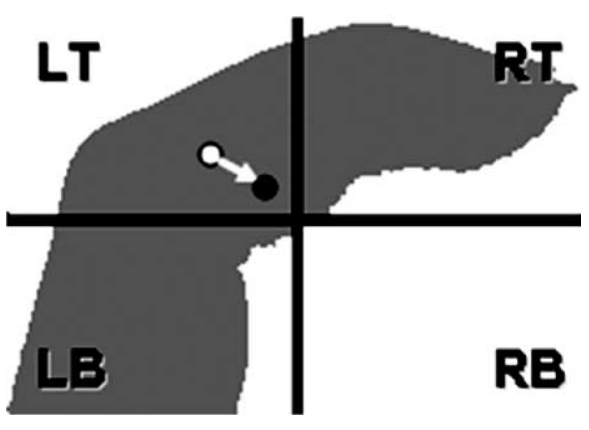

Fig. 10. Illustration of trajectory estimation scheme. The area of extracted object is represented in grey colour.

follow:

$X_{B_{-} O}(t)=\left(\sum_{p \subset S_{O} \cap \text { Part }} x_{p}\right) / \| p \subset S_{O} \cap$ Part $\|$

$Y_{B_{-} O}(t)=\left(\sum_{p \subset S_{O} \cap \text { Part }} y_{p}\right) /\left\|p \subset S_{O} \cap \operatorname{Part}\right\|$

For the example given in Fig. 10, the maximum area can be achieved by summing up RT, LT, and LB. And the LT part plays a corner role in the combination. Therefore, the 'Part' defined in Eqs. (24) and (25) is the LT part and $\left(X_{B_{-} O}(t), Y_{B_{-}} O(t)\right)$ is the basis coordinate, which is indicated by the white spot in Fig. 10.

When we obtained the velocity of the desired objects and their basis coordinate, the position $x_{N_{-} O}(t+1)$ and $y_{N_{-} O}(t+1)$ of the next initial growing seeds for segmenting the same objects $O$ in next frame can be obtained from the following formula:

$x_{N_{-} O}(t+1)=X_{B_{-} O}(t)+V x_{O}(t)$

$y_{N_{-} O}(t+1)=Y_{B_{-} O}(t)+V y_{O}(t)$

Therefore, when the $(t+1)$ th video frame has been captured, according to $\left(x_{N_{-} O}(t+1), y_{N_{-} O}(t+1)\right)$, the proposed trajectory estimation scheme can automatically deploys growing seeds in the $(t+1)$ th video frame, as the black spot in Fig. 10, and the white arrow is the moving velocity of the object $O$.

\section{Experimental results}

In this section, nine testing video clips are applied to the proposed algorithm to demonstrate the tracking performance. The parameters used in the proposed algorithm are listed as follows:

$T=25$ in Eq. (15); $\quad \alpha=1$ in Eq. (16);

$\beta=1$ in Eq. (16);

$\eta_{1}=0.5$ in Eq. (17); $\quad \eta_{2}=0.5$ in Eq. (17).

The video clips are classified into three categories, which are static object with deformation, moving object with fixed camera, and moving object(s) with camera movement. The movie video clips used in this work are video streams after compression, which 


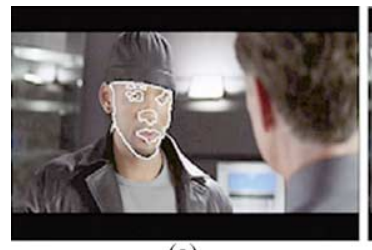

(a)

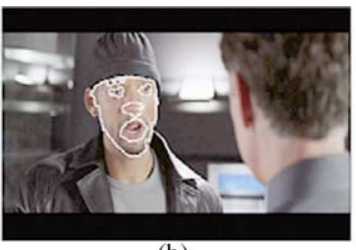

(b)

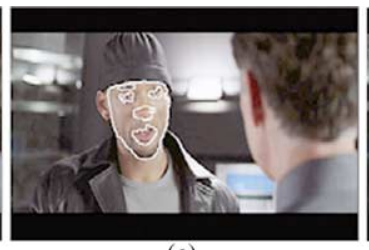

(c)

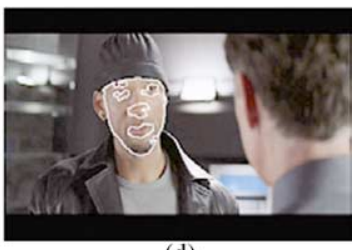

(d)

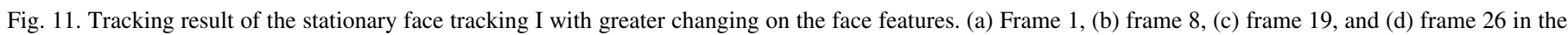
sequence of frames. (The tested video clips were extracted from 'I Robot', issued by $20^{\text {th }}$ Century Fox, 2004.)

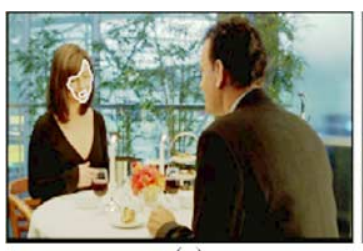

(a)

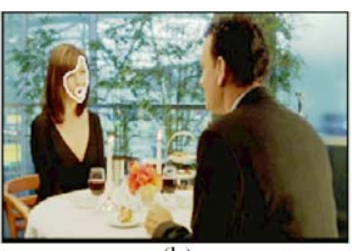

(b)

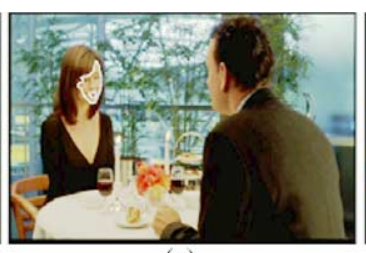

(c)

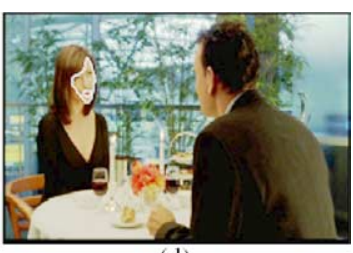

(d)

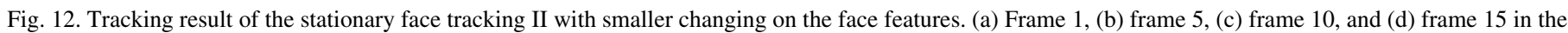
sequence of frames. (The tested video clips were extracted from 'The Terminal', issued by Dreamworks Video, 2004.)

can be utilized to demonstrate the proposed algorithm is robust against noise signals and burred video frames.

\subsection{Static object with deformation}

In this section, we present the evaluation results of the proposed algorithm by tracking a static object in the video frames.

\subsubsection{Video clips 1 from movie: I Robot}

A video stream, captured from the movie called 'I Robot', is used to demonstrate the performance of the proposed tracking algorithm. In this video stream, as shown in Fig. 11, the tracking target is the face of Will Smith. His face was not moving, but because Will was speaking, the shape of his face is changing at all time. The tracking result shows that the proposed algorithm can successfully track the face of Will Smith.

\subsubsection{Video clips from movie: The Terminal}

A video stream, captured from the movie called 'The Terminal', is used to demonstrate the performance of the proposed tracking algorithm. In the video stream shown in Fig. 12, the tracking target is the face of Catherine Zeta-Jones. Similar to the example given in Section 5.2.2, Catherine's face was not moving, but because she was speaking, therefore, the shape of her face is continuously changing. The tracking result shows that the proposed algorithm can precisely generate region masks for tracking the face.

\subsubsection{Video clips 2 from movie: I Robot}

Another video stream shown in Fig. 13, captured from the movie called 'I Robot', is used to evaluate the performance of the proposed tracking algorithm. In this video stream, the tracking target is the chest of robot. The position of the chest of the robot was not changing, but the size of the object was increasing frame to frame. The tracking result shows that the proposed algorithm

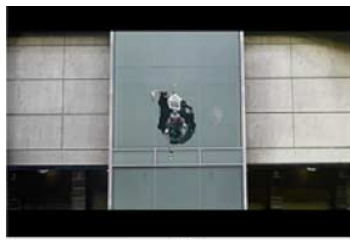

(a)

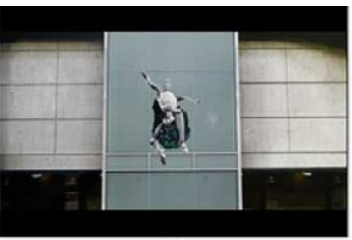

(b)

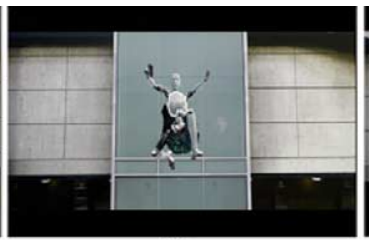

(c)

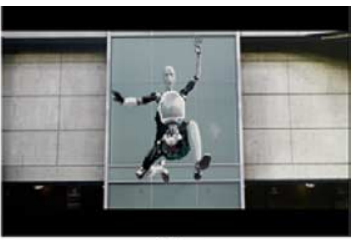

(d)

Fig. 13. Tracking an object with changing area. (a) Frame 1, (b) frame 8, (c) frame 16, and (d) frame 24 in the sequence of frames. (The tested video clips were extracted from 'I Robot', issued by $20^{\text {th }}$ Century Fox, 2004.)

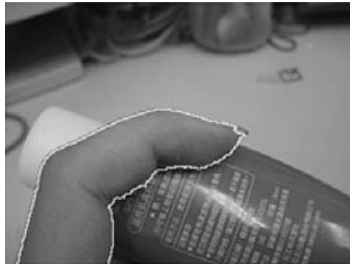

(a)

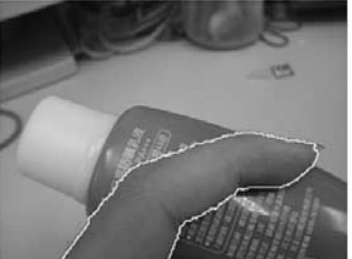

(b)

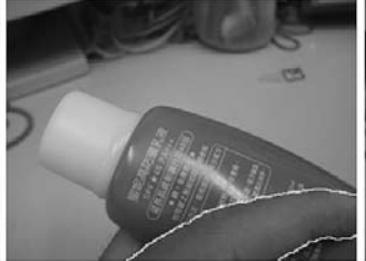

(c)

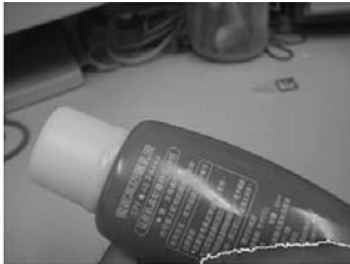

(d)

Fig. 14. Finger moving in complex background. (a) Frame 1, (b) frame 10, (c) frame 20, and (d) frame 30 in the sequence of frames. 
can precisely produce the region masks for tracking the object when the size of the object is frequently changing.

\subsection{Moving object( $(s)$ with stationary camera}

In this section, experiments of moving object with stationary camera are utilized to show the performance of the proposed tracking algorithm for tracking a moving object by a stationary camera.

\subsubsection{Tracking object in complex background 1}

A video clip, as shown in Fig. 14, records a finger slide over a bottle, is used to evaluate the tracking performance of the proposed algorithm. The tracking target is the complete region of the finger. Although the bottle has a lot of Chinese words printed and light reflection on it, the proposed algorithm still tracks the finger very precisely.

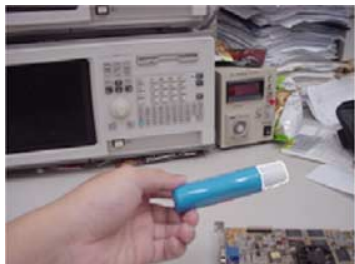

(a)

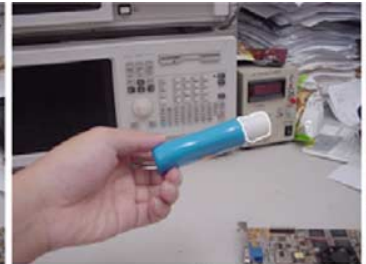

(b)

\subsubsection{Tracking object in complex background 2}

Fig. 15 shows another colour video clip that records a hand taking off a bottle from the desk, which is used to evaluate the tracking performance of the proposed algorithm. The tracking target in this video clip is the cap of the bottle. As shown in the figure, the region of the cap moves through the complex background that consists of many instruments. In this test, the proposed algorithm is still able to successfully track the cap, and the tracking performance has not been influenced by the complex background in the video stream.

\subsubsection{Video clips 3 from movie: I Robot}

The last video stream, captured from the movie called 'I Robot', is used to evaluate the performance of the proposed tracking algorithm. In this video stream, as shown in Fig. 16, the tracking target is the head of a robot. Because both of the robots are fighting to each other, the position of the head of the robot was changing at high speed. The tracking result shows

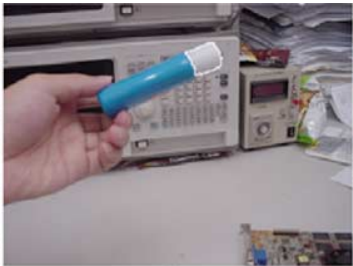

(c)

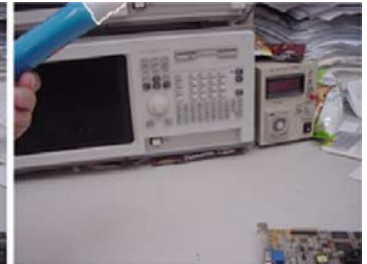

(d)

Fig. 15. Bottle moving in complex background. (a) Frame 1, (b) frame 15, (c) frame 30, and (d) frame 45 in the sequence of frames.

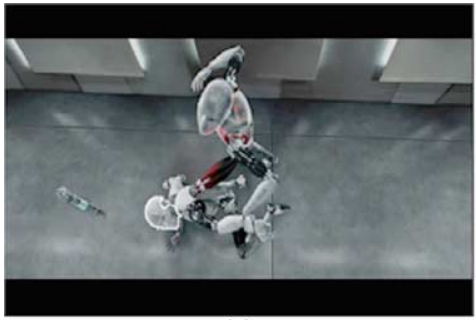

(a)

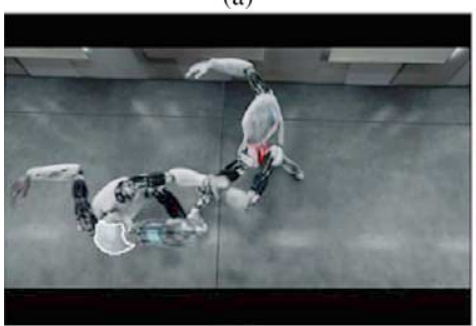

(d)

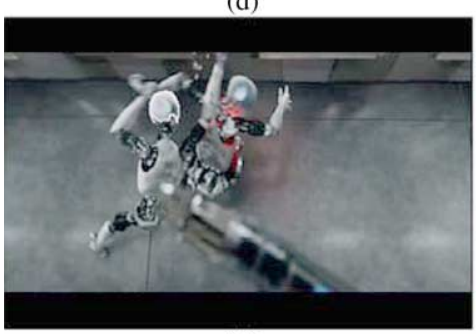

(g)

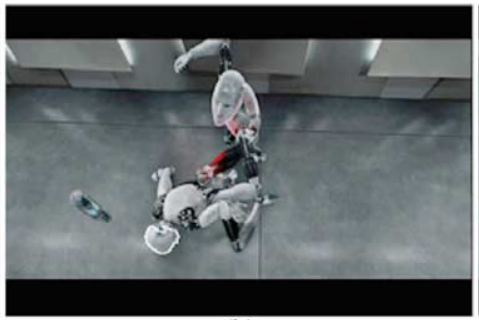

(b)

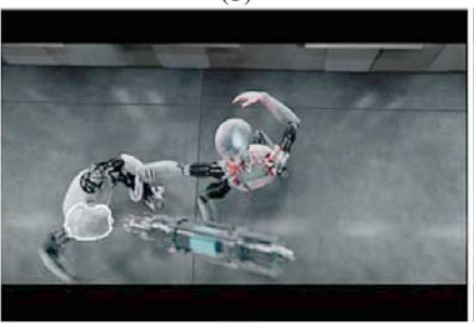

(e)

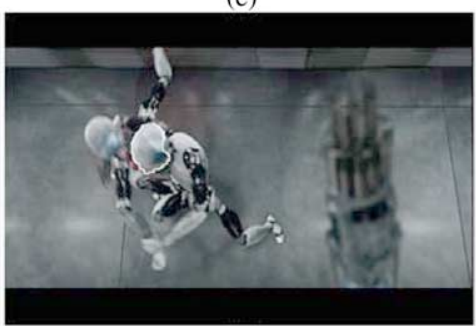

(h)

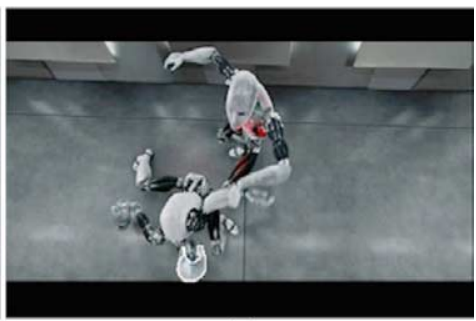

(c)

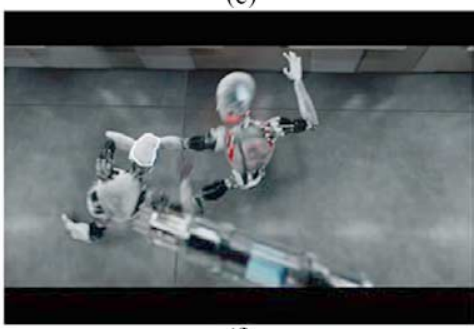

(f)

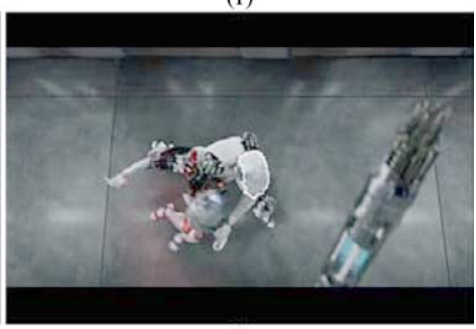

(i)

Fig. 16. Tracking an object at high moving velocity. (a) Frame 1, (b) frame 16, (c) frame 32, (e) frame 48, (f) frame 64, (g) frame 80, (h) frame 96, (i) frame 112 and (j) frame 128 in the sequence of frames. (The tested video clips were extracted from 'I Robot', issued by $20^{\text {th }}$ Century Fox, 2004.) 


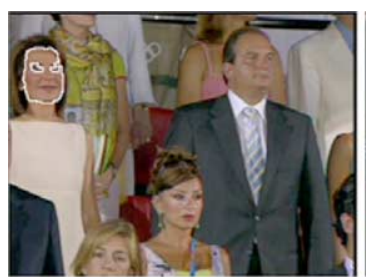

(a)

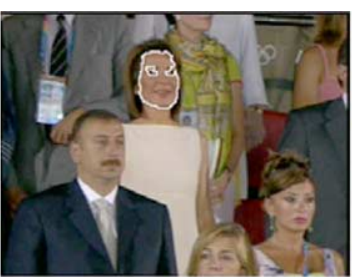

(b)

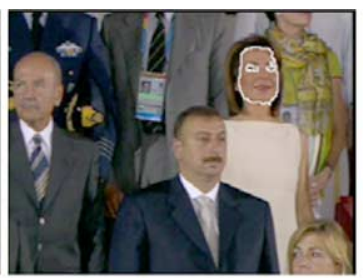

(c)

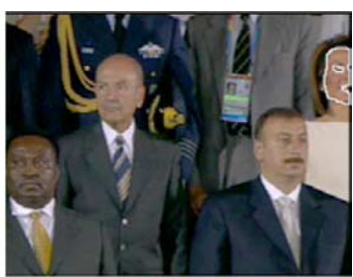

(d)

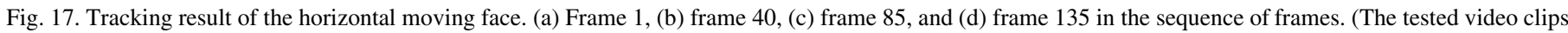
were extracted from 'Olympic Opening Ceremony-Athens 2004', issued by BBC News, 2004.)

that the proposed algorithm is robust against objects movement with high velocity.

\subsection{Moving object(s) with camera movement}

This section demonstrates the performance of the proposed algorithm under tracking one or two moving objects by a mobile camera.

\subsubsection{Video from opening ceremony of Games of the XXVIII Olympiad Athens 2004}

A video stream in Fig. 17 is used to evaluate the tracking performance of the proposed algorithm. The video stream is a part of the TV program that was playing the opening ceremony of Games of the XXVIII Olympiad Athens 2004. The tracking target is the face of Gianna Angelopoulos-Daskalaki, who was the president of the Athens Olympic Organizing Committee (ATHOC). While the Olympiad song was singing, the sense of the camera shifts through Gianna. The tracking result, as depicted in Fig. 17, shows that the proposed tracking algorithm is able to successfully produce the region mask for extracting the face of Gianna.

\subsubsection{Video clip acquired by non-stationary camera}

A sequence of video frames that acquired by a nonstationary camera is used to evaluate the tracking performance of the proposed algorithm. In the video stream, as shown in Fig. 18, a bottle is placed on a desk, and the white cap of the bottle is the target object. Because the video stream is acquired by a non-stationary camera, the background is constantly changing during the tracking process. The tracking result shows that the proposed tracking algorithm is capable of precisely tracking the complete region of a predetermined object, and tracking performance is not influenced by the changing background.

\subsubsection{Multiple objects tracking by non-stationary camera}

In this paragraph, the multiple objects tracking ability of the proposed algorithm is evaluated. A video clip depicted in Fig. 19 records that a hand takes a bottle moving over a silver box. The tracking targets in this example are the cap of the bottle and the silver box. In this video clip, the silver box is placed in a fixed position on the desk, the hand takes the bottle from left side to right side in the sense of the camera, and the sense of the camera is moving from right to left. Therefore, not only the background is constantly changing, but also the two tracking targets are overlapping with each other during the tracking process. The tracking result shows that the proposed algorithm can produce good tracking performance on tracking multiple objects in the video that acquired by a non-stationary camera.

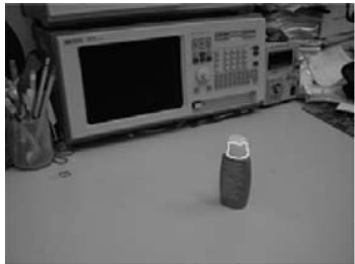

(a)

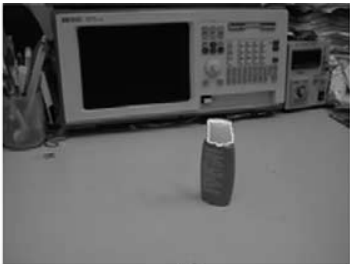

(b)

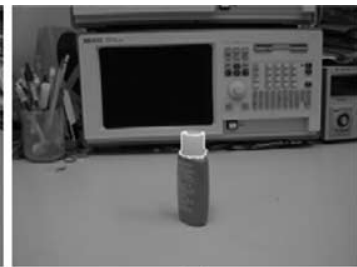

(c)

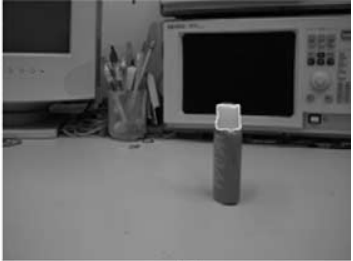

(d)

Fig. 18. Non-stationary camera tracking. (a) Frame 1, (b) frame 15, (c) frame 30, and (d) frame 45 in the sequence of frames.

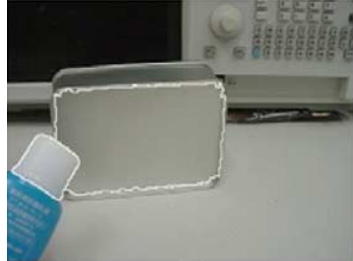

(a)

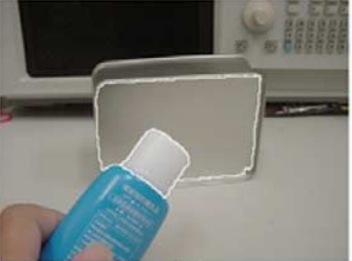

(b)

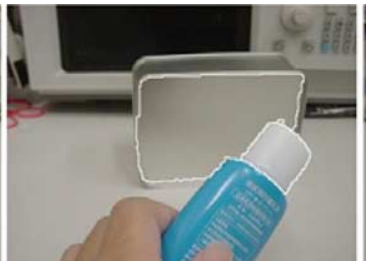

(c)

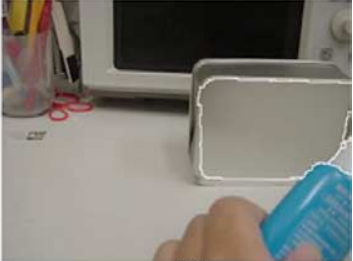

(d)

Fig. 19. Multiple objects tracking. (a) Frame 1, (b) frame 15, (c) frame 30, and (d) frame 45 in the sequence of frames. 


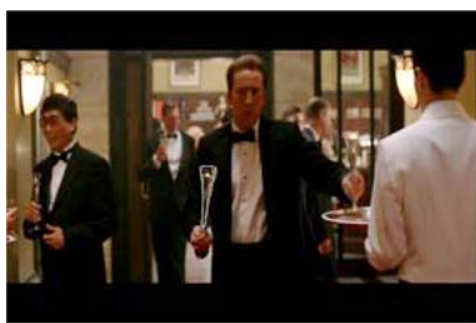

(a)

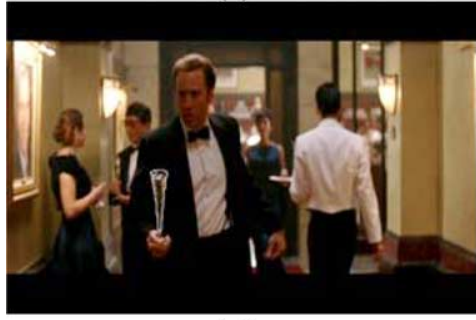

(d)

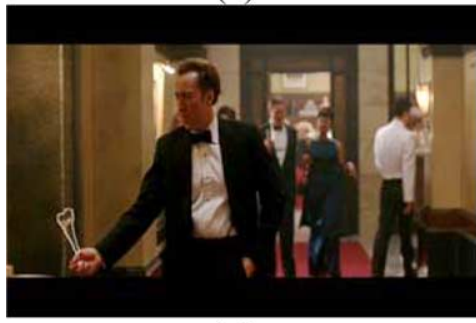

(g)

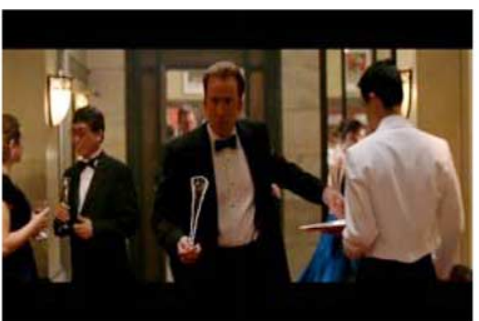

(b)

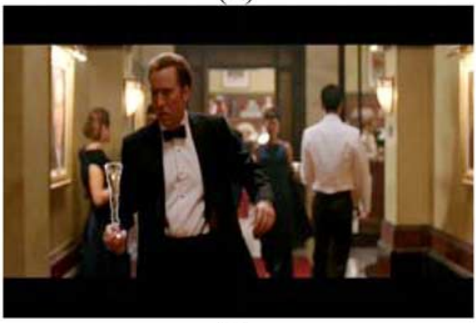

(e)

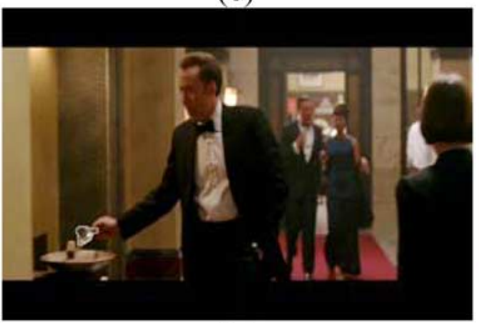

(h)

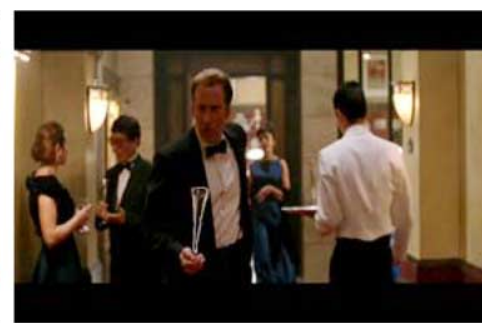

(c)

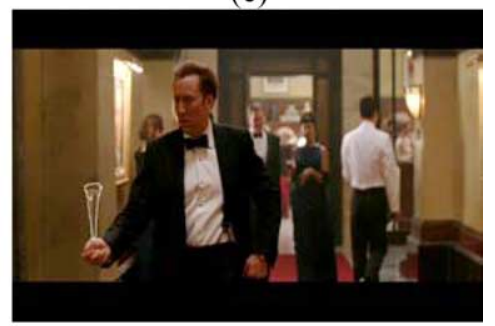

(f)

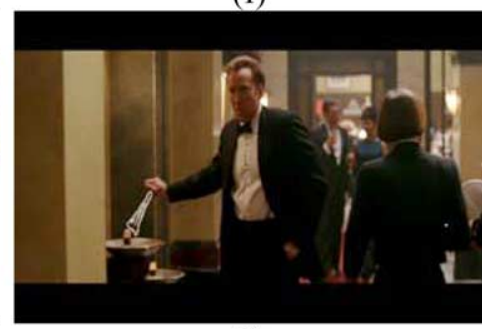

(i)

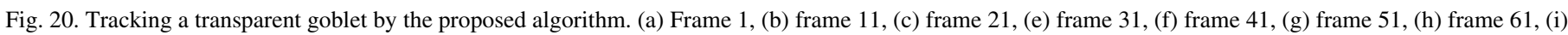

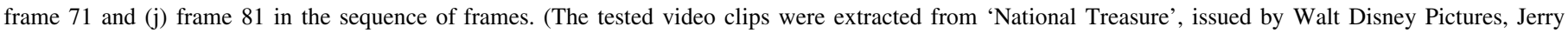
Bruckheimer Films, Junction Entertainment, and Saturn Films, 2004.)

\section{Comparison results}

For comparison, a movie video clip called 'National Treasure', is captured for evaluating the performance of the proposed tracking algorithm. The utilized video clip is shown in Fig. 20, in which Nicolas Cage was walking with a transparent goblet. He dumped wines filled in the goblet in this video clip, and the tracking target is the transparent goblet. The proposed tracking algorithm is applied to this video clip to investigate the tracking performance. Meanwhile, an improved version of Comaniciu's mean-shift approach [4], called adaptive mean-shift approach [14] is also used as a comparative reference for the proposed tracking algorithm. All parameters used in this experiment were the same as listed in Section 5. Since we need to deal with objects with changing shape, the scale adaptation of the adaptive mean-shift approach was settled to about $20 \%$.

The tracking results of the proposed tracking approach are depicted in Fig. 20. We can see that the proposed algorithm is capable of accurately extracting the region of the transparent goblet, and constantly changing orientation and shape of the goblet did not affect the tracking results. On the other hand, the tracking results of the adaptive mean-shift approach are shown in Fig. 21. It is clearly seen that the latter approach can produce a scale-adaptive mask to extract the tracking target, however, the mask also contains many unwanted background information due to the shape of the mask is fixed. Moreover, because the colour of the transparent goblet became insignificant in the 47th frame, the tracking mask was missleaded to a wrong region, and kept tracking an undesired region since then. In this experiment, the proposed algorithm shows good tracking results with robustness to insignificant tracking object. This feature is mainly accomplished by the newly developed edge detector utilized in this work.

\section{Conclusions}

A hybrid approach that combined with mathematical morphology, region growing and merging, and trajectory estimation scheme, is proposed. At first, user can predetermine the desired objects as tracking targets, and then the video frame is processed by the mathematical morphology edge detector to sketch the contour the frame. The contour features are then processed by the segmentation algorithm proposed in Section 3 , which consists of region growing and region merging processes. According to the contour features of the extracted object, we utilized the proposed trajectory estimation scheme for automatically deploying the growing seeds for tracking the same object in further frames. The experimental results show that the tracking performance of the proposed algorithm is pretty good, and the proposed tracking algorithm is also robust against objects occlusion, noise signals and burred video frames. 


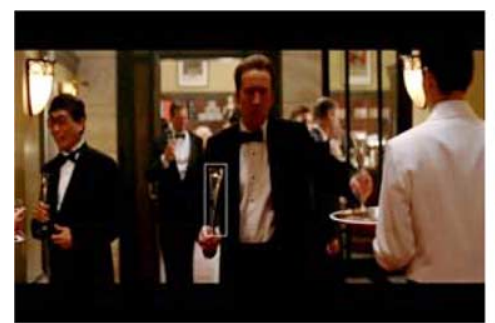

(a)

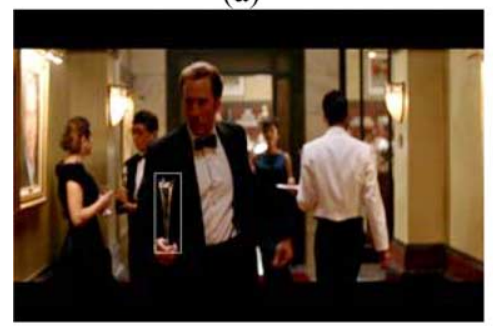

(d)

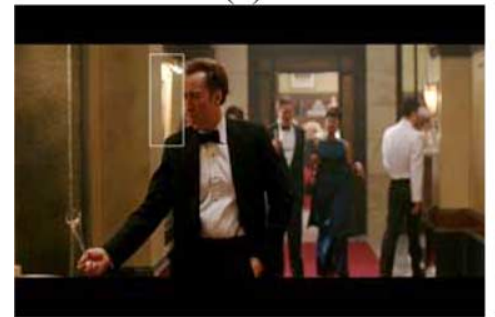

(g)

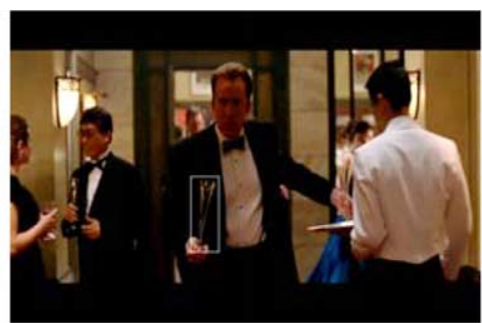

(b)

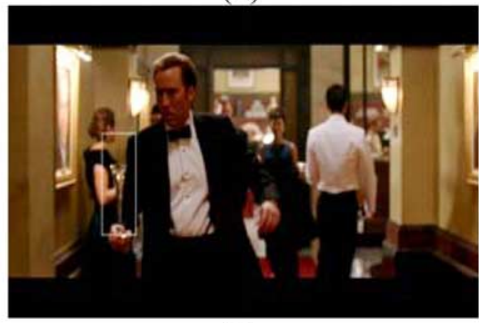

(e)

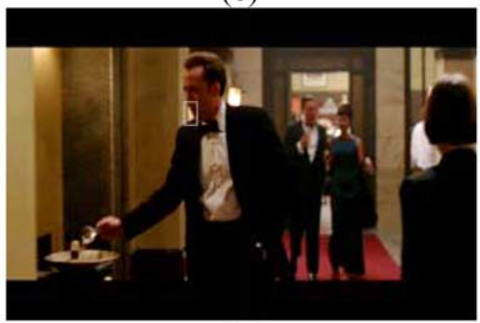

(h)

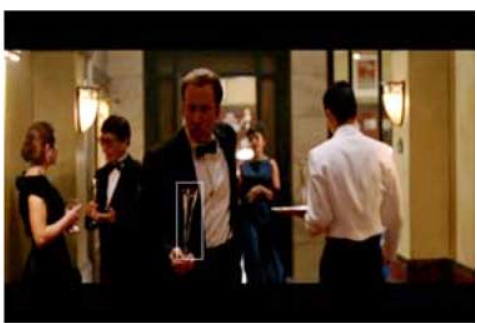

(c)

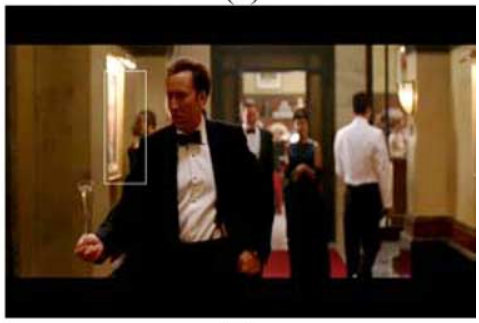

(f)

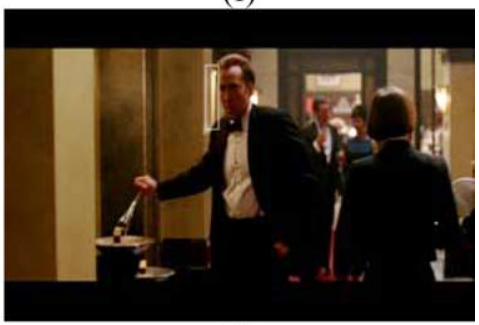

(i)

Fig. 21. Tracking a transparent goblet by the adaptive mean-shift approach [14]. (a) Frame 1, (b) frame 11, (c) frame 21, (e) frame 31, (f) frame 41, (g) frame 51, (h) frame 61, (i) frame 71 and (j) frame 81 in the sequence of frames. (The tested video clips were extracted from 'National Treasure', issued by Walt Disney Pictures, Jerry Bruckheimer Films, Junction Entertainment, and Saturn Films, 2004.)

\section{Acknowledgements}

The authors would like to thank the National Science Council of the Republic of China for financially supporting this research under Contract No. NSC 93-2213-E-002-133. In this work, several video streams were captured from commercial movies, and employed to evaluate the performance of the proposed algorithm. The authors like to thank to $20^{\text {th }}$ Century Fox, Dreamworks Video, Walt Disney Pictures, Jerry Bruckheimer Films, Junction Entertainment, and Saturn Films for their excellences in making and producing of those exclusive and high quality movies.

\section{References}

[1] L. Chiariglione, MPEG and multimedia communications, IEEE Transactions on Circuits and Systems for Video Technology (1997) 5-18.

[2] S.-F. Chang, T. Sikora, A. Puri, Overview of the MPEG-7 standard, IEEE Transactions on Circuits and Systems for Video Technology (2001) 688695.

[3] A. Yilmaz, X. Li, M. Shah, Contour-based object tracking with occlusion handling in video acquired using mobile cameras, IEEE Transactions on Pattern Analysis and Machine Intelligence (2004) 1531-1536.

[4] D. Comaniciu, V. Ramesh, P. Meer, Kernel-based object tracking, IEEE Transactions on Pattern Analysis and Machine Intelligence (2003) 564 575 .
[5] A.D. Jepson, D.J. Fleet, T.F. El-Maraghi, Robust online appearance models for visual tracking, IEEE Transactions on Pattern Analysis and Machine Intelligence (2003) 415-422.

[6] C.P. Huang, Real-time image process in the small-size robot soccer system, Master thesis, Tamkang University, Taiwan, 2002.

[7] A. Yezzi, L. Zollei, T. Kapur, A variational framework for joint segmentation and registration, Proceedings of the Workshop Mathematica Methods in Biomedical Image Analysis (2001) 56-61.

[8] J. Rittscher, A. Blake, A probabilistic background model for tracking, Proceedings of the Europen Conference Computer (2000).

[9] L. Patras, E.A. Hendriks, R.L. Lagendijk, Video segmentation by MAP labeling of watershed segments, IEEE Transactions on Pattern Analysis and Machine Intelligence (2001) 326-332.

[10] Y.T. Hsiao, C.L. Chuang, S.H. Yen, H.J. Lin, A mathematical morphological approach to thin edge detection in dark region, Proceedings of the 4th IEEE International Symposium on Signal Processing and Information Technology (2004) 310-313.

[11] T.H. Hong, A. Rusenfeld, Compact region extraction using weighted pixel linking in a pyramid, IEEE Transactions on Pattern Analysis and Machine Intelligence (1984) 222-229.

[12] L. Leonardis, A. Gupta, R. Bajcsy, Segmentation of range images as the search for geometric parametric models, International Journal of Computer Vision (1995) 253-277.

[13] J. Serra, Image Analysis and Mathematical Morphology, Academic Press, New York, 1982.

[14] D. Comaniciu, V. Ramesh, P. Meer, Real-Time tracking of Non-Rigid objects using mean shift, Proceedings of IEEE Conference on Computer Vision and Pattern Recognition (2000) 142-149. 